\title{
DESEMPENHO DE FRANGOS DE CORTE CRIADOS SOBRE DOIS TIPOS DE CAMA E DUAS DENSIDADES
}

\author{
PERFORMANCE OF CHICKENS CREATED ON TWO TYPES OF BED AND TWO \\ DENSITIES
}
RENDIMIENTO DE POLLOS CREADOS EN DOS TIPOS DE CAMA Y DOS DENSIDADES

Maria Paula Balduino Jorge ${ }^{* 1}$, Roberta Gomes Marçal Vieira Vaz ${ }^{2}$, Mônica Calixto da Silva $^{3}$, Hérica de Araujo Costa ${ }^{4}$, Latóya de Sousa Bezerra ${ }^{4}$, Mayara da Cruz Ribeiro ${ }^{5}$, Aleane Francisca Cordeiro Barbosa ${ }^{5}$, Josimar Santos de Almeida ${ }^{4}$, Jerry Kleuber Felix Monteiro Junior $^{4}$, Magna Ferreira de Oliveira ${ }^{4}$.

\author{
${ }^{1}$ Acadêmica do curso de Zootecnia, Universidade Federal do Tocantins, Araguaína-TO, Brasil. \\ ${ }^{2}$ Professores do Curso de Graduação em Zootecnia, Universidade Federal do Tocantins, Araguaína-TO, Brasil \\ ${ }^{3}$ Professora do Curso de Graduação em Zootecnia, Universidade Federal Rural da Amazônia, Paragominas-PA, Brasil \\ ${ }^{4}$ Pós-graduandos em Ciência Animal Tropical, Universidade Federal do Tocantins, Araguaína-TO, Brasil \\ ${ }^{5}$ Doutoras em Ciência Animal Tropical, Universidade Federal do Tocantins, Araguaína-TO, Brasil \\ *Correspondência: Universidade Federal do Tocantins, Escola de Medicina Veterinária e Zootecnia, Zona Rural, \\ Br 153, Araguaína, Tocantins, Brasil.CEP:77800-000.E-mail nepanac@gmail.com
}

Artigo recebido em 13/03/2020 aprovado em 06/11/2020 publicado em 01/12/2020.

\section{RESUMO}

Objetivou-se avaliar o desempenho produtivo de frangos de corte criados sobre dois tipos de cama e duas densidades. As aves foram distribuídas em delineamento experimental inteiramente casualizado, em arranjo fatorial 2x2, com quatro tratamentos e seis repetições. Foram avaliados o desempenho produtivo, o rendimento de carcaça, os cortes nobres, as vísceras comestíveis, o peso e comprimento do intestino delgado, a gordura abdominal e a qualidade física da carne do peito. Observou-se que os tipos de cama não influenciaram o consumo de ração, o ganho de peso e o peso corporal. Havendo efeito sobre a conversão alimentar. As diferentes densidades influenciaram o consumo de ração e o ganho de peso, sem efeito sobre a conversão alimentar e o peso corporal. As densidades e os tipos de cama não influenciaram os rendimentos de carcaça, coxa, sobrecoxa, as vísceras comestíveis, o peso e comprimento do intestino delgado e a qualidade física da carne do peito. No entanto, o rendimento de peito foi influenciado pela densidade e a gordura abdominal foi influenciada pelo tipo de cama. Conclui-se que a cama de maravalha e a densidade de $24 \mathrm{~kg} / \mathrm{m}^{2}$, apresentam os melhores resultados de desempenho para frangos de corte aos 42 dias de idade. Palavras-chave: frango de corte; maravilhoso; palha de arroz

\section{ABSTRACT}

The objective was to evaluate the productive performance of broilers raised on two types of litter and two densities. The birds were distributed in a completely randomized design, in a $2 \times 2$ factorial arrangement, with four treatments and six replications. Productive performance, carcass yield, noble cuts, edible viscera, small intestine weight and length, abdominal fat and the physical quality of the breast meat were evaluated. It was observed that the types of bed did not influence feed consumption, weight gain and body weight. There is an effect on feed conversion. Different densities influenced feed intake and weight gain, with no effect on feed conversion and body weight. The densities and types of litter did not influence the yields of carcass, thigh, drumstick, edible viscera, the weight and length of the small intestine and the physical quality of the breast meat. However, breast performance was influenced by density and abdominal fat was influenced by the type of bed. It is concluded that the wood shavings and the density of $24 \mathrm{~kg} / \mathrm{m}^{2}$, present the best performance results for broilers at 42 days of age.

Keywords: broiler poultry; marvelous; rice straw 


\section{RESUMEN}

El objetivo fue evaluar el rendimiento productivo de los pollos de engorde criados en dos tipos de basura y dos densidades. Las aves se distribuyeron en un diseño completamente al azar, en un arreglo factorial $2 x 2$, con cuatro tratamientos y seis repeticiones. Se evaluó el rendimiento productivo, el rendimiento de la canal, los cortes nobles, las vísceras comestibles, el peso y la longitud del intestino delgado, la grasa abdominal y la calidad física de la carne de pechuga. Se observó que los tipos de cama no influían en el consumo de alimento, el aumento de peso y el peso corporal. Hay un efecto en la conversión alimenticia. Las diferentes densidades influyeron en la ingesta de alimento y el aumento de peso, sin ningún efecto sobre la conversión alimenticia y el peso corporal. Las densidades y los tipos de basura no influyeron en el rendimiento de la canal, el muslo, el muslo, las vísceras comestibles, el peso y la longitud del intestino delgado y la calidad física de la carne de pechuga. Sin embargo, el rendimiento de los senos fue influenciado por la densidad y la grasa abdominal fue influenciada por el tipo de cama. Se concluye que las virutas de madera y la densidad de $24 \mathrm{~kg} / \mathrm{m}^{2}$ presentan los mejores resultados de rendimiento para los pollos de engorde a los 42 días de edad.

Descriptores: pollo de engorde; maravilloso paja de arroz

\section{INTRODUÇÃO}

As melhorias nas instalações e nos equipamentos na avicultura brasileira, contribuíram para o crescimento da produção de frangos de corte, pois, os produtores passaram a aumentar a quantidade de aves alojadas por metros quadrados. No entanto, para esses que avanços ocorram, as condições do ambiente, no qual, as aves estão submetidas é importante, para que consigam expressar seu máximo desempenho (GOPINGER et al., 2015; HENRIQUE et al., 2017).

Assim, a densidade de criação tem o objetivo de aumentar a produção de carne por metros quadrados, com a finalidade de obter um maior lucro no final da produção. Todavia, maiores densidades, podem piorar o desempenho e reduzir a qualidade da cama, devido as maiores quantidades de excrementos, ocasionando compactação, decorrente do aumento da umidade, o que pode intensificar o surgimento de lesões na carcaça (HENRIQUE et al., 2017).

No entanto, Ravindran et al. (2006) verificaram a influência da densidade de criação (16, 20 e 24 aves $/ \mathrm{m}^{2}$ ) no desempenho, características de carcaça de frangos de corte e observaram que as densidades não promoveram efeito no ganho de peso e no consumo de ração das aves aos 28 e 35 dias. Porém, Buijs et al. (2009) afirmaram que o aumento da densidade $(6,15$,
$23,33,35,41,47$ e $56 \mathrm{~kg} / \mathrm{m}^{2}$ ) afetou negativamente a saúde das pernas, coxim plantar e os joelhos das aves.

Além da densidade, o material utilizado como cama e o manejo utilizado, são fatores importantes dentro do sistema de criação de frangos de corte, pois, podem influenciar tanto no desempenho zootécnico das aves, como nas características de carcaça (BILGILI et al., 2009). A escolha do material que será utilizado para forrar o piso dos aviários, deve levar em consideração a qualidade, a capacidade de absorção, a maciez, não ser tóxico e que libere pouca quantidade de poeira, evitando a incidência de lesões na carcaça, além de proporcionar melhor conforto para as aves (BRITO et al., 2016; DINIZ et al., 2014).

O material normalmente utilizado como cama na criação de frangos de corte, tem sido a maravalha de madeira, entretanto, devido à dificuldade na aquisição desse material, principalmente, ao custo elevado, tem-se utilizado outros substratos, como a casca de arroz, material que possui características semelhante à maravalha (GARCIA et al., 2011). Diante do exposto, objetivou-se avaliar o desempenho produtivo de frangos de corte criados sobre dois tipos de cama e duas densidades.

\section{MATERIAIS E MÉTODOS}


O experimento foi conduzido no Setor de Avicultura da Escola de Medicina Veterinária e Zootecnia da Universidade Federal do Tocantins, localizado em Araguaína - TO.

Foram utilizados 216 pintos de corte, mistos, de um dia de idade, da linhagem Cobb $500^{\circledR}$, com peso inicial médio de $54 \mathrm{~g} \pm 3,37 \mathrm{~g}$. As aves foram homogeneizadas e distribuídas em delineamento experimental inteiramente casualizado (DIC), em arranjo fatorial $2 \times 2$, dois tipos de cama (maravalha e casca de arroz) e duas densidades ( 24 e $30 \mathrm{~kg} / \mathrm{m}^{2}$ ), com quatro tratamentos (casca de arroz e $24 \mathrm{~kg} / \mathrm{m}^{2}$, casca de arroz e $30 \mathrm{~kg} / \mathrm{m}^{2}$, maravalha e $24 \mathrm{~kg} / \mathrm{m}^{2}$ e de maravalha e $30 \mathrm{~kg} / \mathrm{m}^{2}$ ) e seis repetições.

As aves foram alojadas em galpão experimental, provido de 24 boxes de $2,0 \mathrm{~m}^{2}$, coberto com telhas sanduíches e cortinas laterais, manejadas de acordo com o comportamento das aves, cada box possuía um comedouro tubular e um bebedouro automático tipo copo. O manejo da cama foi realizado diariamente com o revolvimento total da cama, sempre nos horários compreendidos entre às 08:00 e às 09:00 da manhã. A espessura da cama adotada foi de $5 \mathrm{~cm}$ de altura. $\mathrm{O}$ abastecimento dos comedouros e a limpeza dos bebedouros foram realizados duas vezes por dia, visando garantir o livre acesso à água e as rações durante todo o período experimental.

Até o $14^{\circ}$ dia de vida, as aves foram aquecidas artificialmente, utilizando-se lâmpadas incandescentes $(60 \mathrm{~W})$, instaladas no interior de todos os boxes. O programa de luz adotado foi o contínuo (24 horas de luz, natural + artificial). As condições ambientais no interior das instalações, durante $\mathrm{o}$ período experimental, foram monitoradas e registradas diariamente a cada 30 minutos, utilizando-se Data Loggers da marca HOBO ware OnSet ${ }^{\circledR}$ Versão 3.4.1, colocados à meia altura dos boxes, possibilitando a obtenção dos valores médios da temperatura do ar, da umidade relativa do ar e da temperatura de globo negro, sendo estes valores convertidos em ITGU (Índice de Temperatura Globo e Umidade), de acordo com Buffington et al. (1981). As dietas experimentais foram calculadas considerando as exigências nutricionais das aves, de acordo com as recomendações de Rostagno et al. (2017), (Tabela 1) nas fases de 1 a 7, de 8 a 21 e de 22 a 42 dias de idade.

As variáveis avaliadas foram o consumo de ração (CR), ganho de peso (GP), conversão alimentar (CA), rendimento de carcaça (RC), rendimentos de cortes nobres (coxa, sobrecoxa e peito), as vísceras comestíveis (coração, fígado, moela), peso e/ou comprimento do intestino delgado, gordura abdominal e a qualidade física da carne do peito (coloração, perda de peso por cozimento e a força de cisalhamento).

As aves foram pesadas no início e no final do período experimental, para determinação do GP. O CR foi calculado considerando a quantidade de ração fornecida e as sobras nos comedouros. A CA foi obtida pela razão entre o consumo de ração ingerido e o ganho de peso das aves, durante o período experimental.

Aos 42 dias de idade, duas aves de cada parcela, com peso corporal próximo ao da média da parcela ( \pm $5 \%$ ), foram submetidas a jejum alimentar de 8 horas e abatidas por deslocamento cervical. Em seguida, foram submetidas aos procedimentos de sangria, escalda, depena e evisceração, para avaliação dos pesos das carcaças inteiras (com pés, pescoço e cabeça) e dos cortes nobres (coxa, sobrecoxa e peito

As vísceras comestíveis, a gordura abdominal e o intestino delgado foram coletados durante a evisceração, limpos, secos em papel toalha e pesados separadamente em balança de precisão. Da moela, foram removidas toda a gordura aderida, seu conteúdo e a membrana coilínea. Além do peso, foram medidos 
o comprimento do intestino delgado do início do duodeno até a junção ileocecal. $\mathrm{O}$ peso relativo da carcaça depenada e eviscerada foram calculados em relação ao peso em jejum. Os pesos relativos dos cortes, das vísceras comestíveis e do intestino delgado foram obtidos em relação à carcaça depenada e eviscerada.

$\mathrm{Na}$ carne crua do peito de frango (sem osso, pele, ligamentos e gordura) foram avaliados a coloração pelo sistema CIELAB $\left(\mathrm{L}^{*}=\right.$ Luminosidade, $\mathrm{a}^{*}=$ teor de vermelho e $b^{*}=$ teor de amarelo) com colorímetro $\left(\right.$ Chroma meter $\left.{ }^{\circledR}\right)$, sendo as leituras realizadas em três pontos distintos, para obtenção da média. A determinação da perda de peso por cocção e da força de cisalhamento foram realizadas de acordo com a metodologia adaptada de Froning e Uijttenboogarte (1988).

Tabela 1 Composição nutricional das dietas para frangos de corte em diferentes fases de criação

\begin{tabular}{|c|c|c|c|}
\hline \multirow[b]{2}{*}{ Ingredientes } & \multicolumn{3}{|c|}{ Fases de criação (dias) } \\
\hline & 1 a 7 & 8 a 21 & 22 a 42 \\
\hline Milho $7,88 \%$ & 56,05 & 58,05 & 62,66 \\
\hline Farelo de Soja (45\%) & 37,18 & 34,45 & 30,55 \\
\hline Óleo de soja & 2,11 & 3,09 & 3,46 \\
\hline Fosfato Bicálcico & 1,82 & 1,67 & 1,17 \\
\hline Calcário & 1,11 & 0,98 & 0,83 \\
\hline Sal comum & 0,51 & 0,50 & 0,44 \\
\hline DL-Metionina & 0,37 & 0,38 & 0,24 \\
\hline L-Lisina & 0,31 & 0,34 & 0,20 \\
\hline L-Treonina & 0,14 & 0,14 & 0,05 \\
\hline Suplemento mineral e vitamínico ${ }^{1 ; 2}$ & 0,40 & 0,40 & 0,40 \\
\hline Total & 100,00 & 100,00 & 100,00 \\
\hline \multicolumn{4}{|c|}{ Composição nutricional calculada } \\
\hline $\mathrm{EM}(\mathrm{kcal} / \mathrm{kg})$ & 2975 & 3050 & 3175 \\
\hline Proteína bruta $(\%)$ & 22,20 & 20,80 & 19,57 \\
\hline Cálcio $(\%)$ & 0,97 & 0,87 & 0,69 \\
\hline Fósforo Disponível (\%) & 0,46 & 0,41 & 0,33 \\
\hline Lisina Digestível (\%) & 1,3 & 1,25 & 1,06 \\
\hline Metionina + cistina Digestível $(\%)$ & 0,96 & 0,92 & 0,79 \\
\hline Metionina Digestível (\%) & 0,65 & 0,64 & 0,50 \\
\hline Treonina Digestível (\%) & 0,86 & 0,82 & 0,70 \\
\hline Sódio (\%) & 0,22 & 0,21 & 0,20 \\
\hline
\end{tabular}

${ }^{1}$ Composição/tonelada fase inicial: Ácido Fólico 150,00 mg, Cobalto 178,00 mg, Cobre 2.675,00 mg, Colina 120,00 g, Colistina 2.000,00 mg, Ferro 11,00 g, Iodo 535,00 mg, Manganês 31,00 g, Matéria mineral 350,00 g, Niacina 7.200,00 mg, Nicarbazina 24,00 g, Pantotenato de Cálcio 2.400,00 mg, Selênio 60,00 mg, Vitamina A 1.920.000,00 UI, Vitamina B1 300,00 mg, Vitamina B12 3.600,00 mg, Vitamina B2 1.200,00 mg, Vitamina B6 450,00 mg, Vitamina D3 360.000,00 UI, Vitamina E 3.600,00 UI, Vitamina H 18,00 mg, Vitamina K 480,00 mg, Zinco 22,00 g. ²Composição/tonelada fase de crescimento: Ácido Fólico 120,00 mg, Cobalto 179,00 mg, Cobre 2.688,00 mg, Colina 108,00 g, Ferro 11,00 g, Iodo 537,00 mg, Lincomicina 800,00 mg, Manganês 31,00 g, Matéria mineral 350,00 g, Niacina 6.000,00 mg, Pantotenato de Cálcio 1.920,00 mg, Salinomicina 12,00 g, Selênio 54,00 mg, Umidade 80.00 g, Vitamina A 1.500.000,00 UI, Vitamina B1 300,00 mg, Vitamina B12 2.800,00 mg, Vitamina B2 960,00 mg, Vitamina B6 450,00 mg, Vitamina D3 300.000,00 UI, Vitamina E 3.000,00 UI, Vitamina H 20,00 mg, Vitamina K 480,00 mg, Zinco 22,00. 
Para determinar a força de cisalhamento, foram retiradas amostras dos filés do peito na forma cilíndrica (1,27 cm de diâmetro), as quais foram colocadas com as fibras orientadas no sentido perpendicular às lâminas do aparelho Warner-Bratzler.

Os dados das variáveis avaliadas foram submetidos aos testes de Normalidade (Cramer Von Mises) e Homocedasticidade (Levene). Satisfeitas essas pressuposições, as variáveis foram submetidas à análise de variância. Adicionalmente as médias dos tratamentos foram comparadas pelo teste Tukey, considerando um nível de significância igual ou inferior a 5\%. As análises estatísticas foram realizadas com o auxílio do programa estatístico SISVAR.

\section{RESULTADOS E DISCUSSÃO}

Os valores médios das temperaturas do ar, média, máxima e mínima no interior do galpão, durante o período experimental, foram de $27,2^{\circ} \mathrm{C}, 36,5$ e 17,4 ${ }^{\circ} \mathrm{C}$, respectivamente, sendo a umidade relativa do ar de $88 \%$, equivalendo ao ITGU de 74,6. O valor de ITGU, ficou dentro das recomendações de Menegali et al. (2010) que relataram que valores de ITGU entre 73,6 77,2 e 69,8 - 81,3, respectivamente, são considerados ideais para o conforto térmico de frangos de corte.

Observou-se que os tipos de cama não influenciaram $(\mathrm{P}>0,05)$ o consumo de ração $(\mathrm{CR})$, o ganho de peso (GP) e o peso corporal (PC). Havendo efeito $(P=0,0230)$ sobre a conversão alimentar $(C A)$. As diferentes densidades influenciaram $(\mathrm{P}=0,0001) \mathrm{o}$ consumo de ração e o $(P=0,0001)$ ganho de peso. Não havendo efeito $(\mathrm{P}>0,05)$ sobre a conversão alimentar $\mathrm{e}$ o peso corporal. Houve interação entre a cama e a densidade de criação para o $(\mathrm{P}=0,0001)$ consumo de ração, o $(\mathrm{P}=0,0028)$ ganho de peso, a $(\mathrm{P}=0,0012)$ conversão alimentar e o $(\mathrm{P}=0,0032)$ peso corporal aos 42 dias de idade (Tabela 2).

Os maiores ganhos de pesos e pesos corporais para os frangos criados sobre a cama de maralhava e na densidade de $24 \mathrm{~kg} / \mathrm{m}^{2}$, podem estar associados ao melhor aproveitamento dos nutrientes pelas aves, ocasionado pela menor competição de alimento. A menor conversão alimentar para os frangos criados sobre a cama de maravalha e na densidade de $30 \mathrm{~kg} / \mathrm{m}^{2}$, pode ter sido em função do menor consumo de ração, o que possivelmente, melhorou a eficiência no processo de absorção dos nutrientes, pela redução da taxa de passagem do alimento no trato gastrointestinal, o que aumentou a exposição do alimento para a ação das enzimas digestivas e aos mecanismos de absorção dos nutrientes pela mucosa intestinal (FAGUNDES, 2011).

Tabela 2 Consumo de ração, ganho de peso, conversão alimentar e peso corporal de frangos de corte aos 42 dias de idade

\begin{tabular}{|c|c|c|c|c|c|c|c|c|}
\hline \multirow{2}{*}{ Variáveis } & \multicolumn{2}{|c|}{ Casca de arroz } & \multicolumn{2}{|c|}{ Maravalha } & \multirow{2}{*}{$\mathrm{CV}^{1}$} & \multicolumn{3}{|c|}{ Valor de $\mathrm{P}^{*}$} \\
\hline & 24 & 30 & 24 & 30 & & $\mathrm{CAM}^{2}$ & $\mathrm{DEN}^{3}$ & $\mathrm{CxD}^{4}$ \\
\hline CR $(g)$ & $3993,7 \mathrm{aA}$ & $3648,3 \mathrm{aB}$ & $4240,8 \mathrm{aA}$ & $4007,9 \mathrm{aB}$ & 3,72 & 0,3628 & 0,0001 & 0,0001 \\
\hline GP $(g)$ & $2203,3 \mathrm{aA}$ & $2093,3 \mathrm{aB}$ & $2306,7 \mathrm{aA}$ & $2150,8 \mathrm{aB}$ & 2,65 & 0,3438 & 0,0001 & 0,0028 \\
\hline CA (g/g) & $1,81 \mathrm{aA}$ & $1,86 \mathrm{aB}$ & $1,84 \mathrm{aA}$ & $1,74 \mathrm{bA}$ & 2,60 & 0,0230 & 0,2659 & 0,0012 \\
\hline $\mathrm{PC}(\mathrm{g})$ & $2646,7 \mathrm{aA}$ & $2645,0 \mathrm{aA}$ & $2751,7 \mathrm{aA}$ & $2700,0 \mathrm{aA}$ & 2,18 & 0,3088 & 0,2785 & 0,0032 \\
\hline
\end{tabular}

$* \overline{\mathrm{P}<0,05 ;}{ }^{1}$ Coeficiente de variação $(\%) ;{ }^{2} \mathrm{CAM}=$ cama; ${ }^{3} \mathrm{DEN}=$ densidade; ${ }^{4} \mathrm{CxD}=$ cama x densidade. Médias com letras minúsculas distintas na mesma linha para os tipos de cama e maiúsculas na mesma linha para as densidades, no mesmo parâmetro, diferem a $5 \%$ de probabilidade de erro pelo teste de Tukey. 
Para os rendimentos de carcaça (RC), coxa (RCX) e sobrecoxa (RSCX), observou-se que as aves criadas nas densidades de 24 e $30 \mathrm{~kg} / \mathrm{m}^{2}$, apresentaram respostas semelhantes $(\mathrm{P}>0,05)$, independentemente do tipo de cama que foram submetidas. No entanto, o rendimento de peito (RP) foi influenciado $(\mathrm{P}=0,0216)$ pela densidade, com maior rendimento para as aves criadas sobre a cama de maravalha e a densidade de $24 \mathrm{~kg} / \mathrm{m}^{2}$ (Tabela 3).

Os maiores rendimentos de peito, observados nas densidades de $24 \mathrm{~kg} / \mathrm{m}^{2}$, podem estar relacionados ao maior ganho de peso das aves, criadas sobre essa densidade. De acordo com Nowicki et al. (2011), a linhagem Cobb é considerada rústica e resistente em várias situações, tais como manejo, temperatura, estresse, densidade, além de apresentar maior

Tabela 3 Rendimentos de carcaça (RC), peito (RP), coxa (RCX) e sobrecoxa (RSCX) de frangos de corte aos 42 dias criados sobre dois tipos de cama (palha de arroz e maravalha) e duas densidades $\left(24\right.$ e $\left.30 \mathrm{~kg} / \mathrm{m}^{2}\right)$

\begin{tabular}{|c|c|c|c|c|c|c|c|c|}
\hline \multirow{2}{*}{ Variáveis } & \multicolumn{2}{|c|}{ Palha de arroz } & \multicolumn{2}{|c|}{ Maravalha } & \multirow{2}{*}{$\mathrm{CV}^{1}$} & \multicolumn{3}{|c|}{ Valor de $\mathrm{P}^{*}$} \\
\hline & 24 & 30 & 24 & 30 & & $\mathrm{CAM}^{2}$ & $\mathrm{DEN}^{3}$ & $\mathrm{C} \times \mathrm{D}^{4}$ \\
\hline $\mathrm{RC}(\mathrm{g})$ & $85,94 \mathrm{aA}$ & $86,22 \mathrm{aA}$ & $86,74 \mathrm{Aa}$ & $87,25 \mathrm{aA}$ & 1,59 & 0,1160 & 0,4911 & 0,8391 \\
\hline $\mathrm{RP}(\mathrm{g})$ & $32,33 \mathrm{aA}$ & $30,96 \mathrm{aB}$ & $33,20 \mathrm{a} \mathrm{A}$ & $31,07 \mathrm{aB}$ & 5,4 & 0,4952 & 0,0216 & 0,5881 \\
\hline $\mathrm{RCX}(\mathrm{g})$ & $9,83 \mathrm{aA}$ & $9,80 \mathrm{aA}$ & $9,77 \mathrm{Aa}$ & $9,73 \mathrm{aA}$ & 4,43 & 0,7051 & 0,8626 & 0,9735 \\
\hline $\operatorname{RSCX}(\mathrm{g})$ & $12,71 \mathrm{aA}$ & $12,84 \mathrm{aA}$ & $12,74 \mathrm{Aa}$ & $13,12 \mathrm{aA}$ & 5,81 & 0,6114 & 0,4066 & 0,6748 \\
\hline
\end{tabular}

*P<0,05; ${ }^{1}$ Coeficiente de variação $(\%) ;{ }^{2} \mathrm{CAM}=$ cama; ${ }^{3} \mathrm{DEN}=$ densidade; ${ }^{4} \mathrm{CxD}=$ cama $\mathrm{x}$ densidade; Médias com letras minúsculas distintas na mesma linha para os tipos de cama e maiúsculas na mesma linha para as densidades, no mesmo parâmetro, diferem a $5 \%$ de probabilidade de erro pelo teste de Tukey.

capacidade de deposição muscular, principalmente na região do peito. $\mathrm{O}$ que pode justificar o maior rendimento de peito encontrado no presente estudo.

Observou-se que os pesos relativos das vísceras comestíveis (coração, moela e fígado), o peso e comprimento do intestino delgado, não foram influenciados $(\mathrm{P}>0,05)$ pelas densidades e os tipos de cama. No entanto, o tipo de cama influenciou ( $\mathrm{P}=$ 0,0089) a gordura abdominal, com maiores valores para as aves criadas sobre a cama de palha de arroz (Tabela 4).

De acordo com Murakami et al. (2010), a deposição de gordura abdominal é um problema na produção de carne de frango, pois, parte da gordura depositada é perdida durante a evisceração da carcaça, o que resulta em menor rendimento de carcaça. Todavia, neste estudo, o aumento da gordura abdominal, não foi capaz de influenciar essa variável, o que denota que o padrão de qualidade do rendimento de carcaça foi mantido, sendo uma característica de relevância industrial, por ser um dos cortes de maior valor e interesse para os consumidores.

Observou-se que as diferentes densidades e os tipos de cama não influenciaram $(\mathrm{P}>0,05)$ a coloração da carne do peito $\left(L^{*}=\right.$ luminosidade, $a^{*}=$ vermelho, $\mathrm{b}^{*}=$ amarelo), o pH, a temperatura (TEMP), perda de peso por descongelamento (PPDES), perda de peso por cocção (PPCO) e a força de cisalhamento (FC) dos frangos de corte aos 42 dias de idade (Tabela 5).

Moreira et al. (2004) observaram que diferentes densidades não influenciaram a perda de peso por cocção e a força de cisalhamento. Entretanto, Diniz et al. (2014) avaliaram a cama de maravalha nova e reutilizada e observaram que os tipos de cama não influenciaram a coloração da carne do peito $\left(\mathrm{L}^{*}=\right.$ luminosidade, $a^{*}=$ vermelho, $b^{*}=$ amarelo) e a perda de peso por cocção, mas, verificaram efeito na maciez da carne de frangos de corte.

De modo geral, nota-se que os melhores resultados de desempenho, rendimento de carcaça e 
cortes, foram observados nos frangos criados na cama

de maravalha e na densidade de $24 \mathrm{~kg} / \mathrm{m}^{2}$, sem efeito

para os parâmetros de qualidade da carne.

Tabela 4 Peso relativo das vísceras comestíveis (coração, moela e fígado), gordura abdominal, peso e comprimento do intestino delgado $(\mathrm{m})$ de frangos de corte aos 42 dias

\begin{tabular}{|c|c|c|c|c|c|c|c|c|}
\hline \multirow{2}{*}{ Variáveis } & \multicolumn{2}{|c|}{ Palha de arroz } & \multicolumn{2}{|c|}{ Maravalha } & \multirow{2}{*}{$\mathrm{CV}^{1}$} & \multicolumn{3}{|c|}{ Valor de $\mathrm{P}^{*}$} \\
\hline & 24 & 30 & 24 & 30 & & $\mathrm{CAM}^{2}$ & $\mathrm{DEN}^{3}$ & $\mathrm{C} \times \mathrm{D}^{4}$ \\
\hline Coração (\%) & $0,35 \mathrm{aA}$ & $0,39 \mathrm{aA}$ & $0,38 \mathrm{aA}$ & $0,37 \mathrm{aA}$ & 18,37 & 0,9336 & 0,6382 & 0,3791 \\
\hline Fígado (\%) & $1,79 \mathrm{aA}$ & $1,76 \mathrm{aA}$ & $1,90 \mathrm{aA}$ & $1,81 \mathrm{aA}$ & 13,32 & 0,4573 & 0,5324 & 0,7571 \\
\hline Moela $(\%)$ & $1,18 \mathrm{aA}$ & $1,14 \mathrm{aA}$ & $1,19 \mathrm{aA}$ & $1,18 \mathrm{aA}$ & 12,30 & 0,6823 & 0,7371 & 0,7843 \\
\hline Gordura abdominal (\%) & $1,61 \mathrm{bA}$ & $1,58 \mathrm{bA}$ & $1,22 \mathrm{aA}$ & $1,36 \mathrm{aA}$ & 17,85 & 0,0089 & 0,6343 & 0,4500 \\
\hline Peso intestino $(\%)$ & $2,07 \mathrm{aA}$ & $2,01 \mathrm{aA}$ & $2,04 \mathrm{aA}$ & $2,01 \mathrm{aA}$ & 10,68 & 0,8709 & 0,6246 & 0,8773 \\
\hline Comprimento do intestino & $1,70 \mathrm{aA}$ & $1,72 \mathrm{aA}$ & $1,66 \mathrm{aA}$ & $1,75 \mathrm{aA}$ & 5,69 & 0,9254 & 0,1829 & 0,3924 \\
\hline
\end{tabular}

*P<0,05; ${ }^{1}$ Coeficiente de variação (\%); ${ }^{2} \mathrm{CAM}=$ cama; ${ }^{3} \mathrm{DEN}=$ densidade; ${ }^{4} \mathrm{CxD}=$ cama $\mathrm{x}$ densidade; Médias com letras minúsculas distintas na mesma linha para os tipos de cama e maiúsculas na mesma linha para as densidades, no mesmo parâmetro, diferem a $5 \%$ de probabilidade de erro pelo teste de Tukey.

Tabela 5 Coloração da carne do peito $\left(\mathrm{L}^{*}=\right.$ luminosidade, $\mathrm{a}^{*}=$ vermelho, $\mathrm{b}^{*}=$ amarelo), perda de peso por cocção (PPCO) e a força de cisalhamento (FC) de carne de frangos de corte ao aos 42 dias de idade, criados sobre dois tipos de cama (palha de arroz e maravalha) e duas densidades $\left(24\right.$ e $\left.30 \mathrm{~kg} / \mathrm{m}^{2}\right)$

\begin{tabular}{|c|c|c|c|c|c|c|c|c|}
\hline \multirow{2}{*}{ Variáveis } & \multicolumn{2}{|c|}{ Palha de arroz } & \multicolumn{2}{|c|}{ Maravalha } & \multirow{2}{*}{$\mathrm{CV}^{1}$} & \multicolumn{3}{|c|}{ Valor de $\mathrm{P}^{*}$} \\
\hline & 24 & 30 & 24 & 30 & & $\mathrm{CAM}^{2}$ & $\mathrm{DEN}^{3}$ & $\mathrm{C} \times \mathrm{D}^{4}$ \\
\hline $\mathrm{L}^{*}$ & 60,26 & 60,78 & 62,58 & 61,24 & 2,88 & 0,0682 & 0,5774 & 0,2102 \\
\hline$a^{*}$ & 11,92 & 11,60 & 11,04 & 10,98 & 7,84 & 0,0523 & 0,5983 & 0,7283 \\
\hline$b^{*}$ & 16,09 & 14,31 & 15,15 & 14,85 & 10,21 & 0,7470 & 0,1138 & 0,2530 \\
\hline PPCO (\%) & 21,24 & 18,60 & 20,17 & 19,76 & 13,60 & 0,9674 & 0,1842 & 0,3263 \\
\hline $\mathrm{FC}\left(\mathrm{kgf} / \mathrm{cm}^{2}\right)$ & 1,25 & 1,41 & 1,41 & 1,36 & 22,75 & 0,6605 & 0,6657 & 0,4092 \\
\hline
\end{tabular}

$* \overline{\mathrm{P}}<0,05 ;{ }^{1}$ Coeficiente de variação $(\%) ;{ }^{2} \mathrm{CAM}=$ cama $;{ }^{3} \mathrm{DEN}=$ densidade $;{ }^{4} \mathrm{CxD}=$ cama $\mathrm{x}$ densidade.

\section{CONCLUSÃO}

A cama de maravalha e a densidade de $24 \mathrm{~kg} / \mathrm{m}^{2}$, apresentam os melhores resultados de desempenho para frangos de corte aos 42 dias de idade.

\section{AGRADECIMENTO}

"O presente trabalho foi realizado com o apoio UFT".

Todos os autores declararam não haver qualquer potencial conflito de interesses referente a este artigo.

\section{REFERÊNCIAS}

BILGILI, S.F.; HESS, J.B.; BLAKE, J.P. MACKLIN, K.S.; SAENMAHAYAK, B.; SIBLEY, J.L. Influence of bedding material on footpad dermatitis in broiler chickens. Journal of Applied Poultry Research, Champaign, v. 18, n. 3, p. 583-589, 2009.

BRITO D.A.P.; BRITO. D.R.B.; GOMES A. M.N.; CUNHA.; A. DOS S.; FILHO, U.A.S.; PINHEIRO. A.A. Desempenho produtivo e rendimento de carcaça de frangos criados em diferentes materiais de cama aviária. Ciência Animal Brasileira, Goiânia, v.17, n.2, p. 192-197, 2016.

BUIJS, S.; KEELING, L.; RETTENBACHER, S.; VAN POUCKE, E.; TUYTTENS, F.A.M. Stocking density effects on broiler welfare: Identifying sensitive ranges for different indicators. Poultry Science, Champaign, v. 88, n. 8, p. 1536-1543, 2009.

BUFFINGTON, D.E.; COLLAZOAROCHO, A.; CANTON, G.H.; PITT, D.; THATCHER, W.W.; COLLIER, R.J. Black globe humidity index (BGHI) as comfort equation for dairy cows. Transactions of the ASAE, v.24, n.3, p.711-714, 1981. 
DINIZ T.; DE MELLO T.; MALAGOLI; LOLLI. Effect of environmental temperature and reuse of bed on broiler meat quality. CES Medicina Veterinária e Zootecnia, v. 9, n. 2, p. 218-226, 2014.

FAGUNDES, N.S. Desenvolvimento do sistema digestório e da capacidade digestiva de frangos de corte alimentados com diferentes níveis de energia metabolizável. 2011.

FRONING， G.W. e UIJTTENBOOGARTE， T.G. Effect of post mortem electrical stimulation on color, texture, $\mathrm{pH}$ and cooking loses of hold and cold deboned chicken broiler breast meat. Poultry Science, v. 67, n. 11, p. 1536-1544, 1988.

GARCIA, R.G.; PAZ, I.C.L.A.; CALDARA, F.R. Papel da cama na produção e bem-estar de frangos de corte. Revista Avisite, v. 47, p. 46-50, 2011.

GOPINGER, E.; AVILA, V.S.D.; PERONDI, D.; CATALAN, A. A.D.S.; KRABBE, E.L.; ROLL, V.F.B. Performance, carcass characteristics and litter moisture in broilers housed at two densities. Acta Scientiarum. Animal Sciences, Maringá, v. 37, n. 1, p. 35-39, 2015.

HENRIQUE, C.D.S.; FRÓES, G.O.A.; SILVA, F.T.; SANTOS, S.E.; FINOTTI, F.R.M.; B., DE FREITAS, A.A.; Garcia E.R.M.; GIUSTI, B.L.D. Effect of stocking density on performance, carcass yield, productivity, and bone development in broiler chickens Cobb 500®. Semina: Ciências Agrárias, Londrina, v. 38, n. 4, p. 2705-2718, 2017.

MENEGALI, I.; BAÊTA D.C.F.; TINÔCO, I.D.F.F.; CORDEIRO, M.B.; DE CARVALHO GUIMARÃES, M. C. Desempenho produtivo de frangos de corte em diferentes sistemas de instalações semiclimatizadas no sul do Brasil. Revista engenharia na agriculturareveng, v. 18, n. 6, p. 461-471, 2010.
MURAKAMI, K.T.T.; PINTO, M.F.; PONSANO, E.H.G.; GARCIA NETO, M. Desempenho produtivo e qualidade da carne de frangos alimentados com ração contendo óleo de linhaça. Pesquisa Agropecuária Brasileira, v. 45, n. 4, p. 401-407, 2010.

MOREIRA, J.; MENDES, A.A.; ROÇA, R.D.O.; GARCIA, E.A.; NAAS, I.D.A.; GARCIA, R.G.; PAZ, I.C.L.D.A. Efeito da densidade populacional sobre desempenho, rendimento de carcaça e qualidade da carne em frangos de corte de diferentes linhagens comerciais. Revista Brasileira de Zootecnia, v. 33, n. 6, p. 1506-1519, 2004.

NOWICKI, R.; BUTZGE, E.; OTUTUMI, L.K.; JÚNIOR, R.P.; ALBERTON, L.R.; MERLINI, L.S.; GERÔNIMODA E.; SILVA CAETANO, I.C.L. Desempenho de frangos de corte criados em aviários convencionais e escuros. Arquivos de Ciências Veterinárias e Zoologia da UNIPAR, v. 14, n. 1, p. 25-28, 2011.

OLIVEIRA, R.D.; DONZELE, J.L.; ABREU, M.D.; FERREIRA, R.A.; VAZ, R.G.M.V., CELLA, P.S.A. Efeitos da temperatura e da umidade relativa sobre $\mathrm{O}$ desempenho e o rendimento de cortes nobres de frangos de corte de 1 a 49 dias de idade. Revista Brasileira de Zootecnia, v. 35, n. 3, p. 797-803, 2006.

RAVINDRAN, V.; THOMAS, D.V.; THOMAS, D.G.; MOREL, P.C. Performance and welfare of broilers as affected by stocking density and zinc bacitracin supplementation. Animal Science Journal, Japão, v. 77, n. 1, p. 110-116, 2006.

ROSTAGNO, H.S.; ALBINO, L.F.T.; DONZELE, J.L. GOMES, P.C.; OLIVEIRA, R.F.; LOPES, D.C.; FERREIRA, A.S. Composição de alimentos e exigências nutricionais. Tabelas Brasileiras para aves e suínos. Viçosa, MG: UFV, Departamento de Zootecnia, p. 141, 2017. 